\title{
An anomalous dip in thermoelectric power of $\mathrm{Nd}_{1-x} \mathrm{Pr}_{x} \mathrm{Ba}_{2} \mathrm{Cu}_{3} \mathrm{O}_{7-\delta}$
}

\author{
S.R. Ghorbani *, Ö. Rapp \\ Solid State Physics, Department of Microelectronics and Information Technology, KTH Electrum 229, SE-164 40 Kista, Sweden
}

\begin{abstract}
The thermoelectric power, $S$, has been studied for sintered samples of $\mathrm{Nd}_{1-x} \operatorname{Pr}_{x} \mathrm{Ba}_{2} \mathrm{Cu}_{3} \mathrm{O}_{7-\delta}$ with $0 \leqslant x \leqslant 0.30$ in the temperature range from the superconducting $T_{\mathrm{c}}$ to room temperature. $S$ increases with decreasing temperature, and has a broad maximum at $T^{\text {max }}$ in the region around $120 \mathrm{~K}$ before decreasing strongly when $T_{\mathrm{c}}$ is approached. Several properties indicate a decrease of charge concentration with increasing doping, $x$, from $S(x, 290 \mathrm{~K}), T^{\mathrm{max}}$, and the resistivity $\rho(x, 290 \mathrm{~K})$. An anomaly has been observed in $S(T)$ for $x \geqslant 0.20$ in the form of a dip at $78 \mathrm{~K}$ of order $15 \%$ of $S$. The origin of this feature is not known.
\end{abstract}

(C) 2003 Elsevier Science B.V. All rights reserved.

Keywords: Superconductivity; Thermoelectric power; Pr doping

\section{Introduction}

In the RE-123 ( $\mathrm{RE}=$ rare earth element) superconductors the RE's can be exchanged for each other with only small effect on the critical temperature $T_{\mathrm{c}}$ with the exception of $\operatorname{Pr}$ substitution which decreases $T_{\mathrm{c}}$ dramatically. Different explanations [1-4] for the depression of $T_{\mathrm{c}}$ have been suggested. Varying results for the valence of $\operatorname{Pr}$ is one reason. Recent neutron diffraction results in $\mathrm{Nd}(\mathrm{Pr})-123$ [5] and analysis of $T_{\mathrm{c}}$ in $\mathrm{Y}(\mathrm{Pr})-123$ [6] have suggested that hole localisation in the $\mathrm{Pr}^{4+}$ site is the main reason for the suppression of superconductivity by Pr. The observation of superconductivity in single crystals of $\mathrm{PrBa}_{2} \mathrm{Cu}_{3} \mathrm{O}_{7-\delta}$ [7,8] further complicates the understanding of the role played by Pr in the suppression of $T_{\mathrm{c}}$. In our previous work [5], resistivity measurements in $\mathrm{Nd}_{1-x} \mathrm{Pr}_{x} \mathrm{Ba}_{2} \mathrm{Cu}_{3} \mathrm{O}_{7}$ showed a decreasing metallic behaviour with increased resistivity and decreased slope of the normal state resistivity vs. temperature with increasing $x$. Neutron diffraction data

\footnotetext{
${ }^{*}$ Corresponding author.

E-mail address: ghorbani@ftf.kth.se (S.R. Ghorbani).
}

indicated that the $\mathrm{Cu} 1-\mathrm{O} 4$ and $\mathrm{Cu} 2-\mathrm{O} 4$ distances and the oxygen content were independent of Pr doping in $\mathrm{Nd}(\mathrm{Pr})-123$. Bond valence sum (BVS) calculations showed a constant $\mathrm{Cu} 2$ valence but a decrease of the total hole concentration in the $\mathrm{CuO}_{2}$ plane. To further investigate the effect of $\mathrm{Pr}$ in $\mathrm{Nd}_{1-x} \mathrm{Pr}_{x} \mathrm{Ba}_{2} \mathrm{Cu}_{3} \mathrm{O}_{7-\delta}$ system, we have measured the thermoelectric power $S(x, T)$.

\section{Sample characterisation and experimental}

Samples of $\mathrm{Nd}_{1-x} \mathrm{Pr}_{x} \mathrm{Ba}_{2} \mathrm{Cu}_{3} \mathrm{O}_{7-\delta}$ (with $x=0,0.05$, $0.10,0.15,0.20$ and 0.30 ) were prepared by standard solid-state methods. Starting materials were high purity $\mathrm{Nd}_{2} \mathrm{O}_{3}, \mathrm{BaCO}_{3}, \mathrm{CuO}$, and $\operatorname{Pr}_{6} \mathrm{O}_{11}$. The samples were pressed into pellets and calcinated in air at 900, 920 and $920{ }^{\circ} \mathrm{C}$ with intermediate grindings. They were then annealed in flowing oxygen at $460{ }^{\circ} \mathrm{C}$ for three days and the temperature was finally decreased to room temperature at a rate of $12{ }^{\circ} \mathrm{C} / \mathrm{h}$.

The samples were characterised by X-ray powder diffraction (XRD). The XRD patterns were recorded in a Guinier-Hägg focusing camera using $\mathrm{CuK}_{\alpha}$ radiation with $\mathrm{Si}$ as an internal standard [9]. The XRD results for 
Pr-doped Nd-123 samples displayed single-phase behaviour. All XRD patterns were indexed with an orthorhombic unit cell.

The electrical resistivity was measured with a standard dc four-probe method. Electrical leads were attached to the sample by silver paint and heat treated at $300{ }^{\circ} \mathrm{C}$ in flowing oxygen for half an hour, which gave contact resistances of order 1-2 $\Omega$. Thermoelectric power measurements were made on sintered bars of typical dimensions $0.5 \times 2.5 \times 10 \mathrm{~mm}^{3}$, using a small, reversible temperature difference of $1.5 \mathrm{~K}$.

\section{Results and discussion}

Fig. 1 shows the thermoelectric power $S$ as a function of temperature and $\mathrm{Pr}$ concentration for $\mathrm{Nd}_{1-x} \mathrm{Pr}_{x} \mathrm{Ba}_{2}-$ $\mathrm{Cu}_{3} \mathrm{O}_{7-\delta} . S$ first increases with increasing temperature towards a broad maximum at $T^{\max }$ above the superconducting $T_{\mathrm{c}}$, and then decreases up to room temperature. Both $S$ and $T^{\max }$ increase with increasing $x$. As further illustrated in the inset of Fig. 1, a dip in $S(T)$ was observed at a temperature in the range $78-79 \mathrm{~K}$. This feature only occurred for $x \geqslant 0.20$. The proximity to the boiling point of liquid nitrogen might suggest an experimental artefact from nitrogen boil off. Such an effect appears to be unlikely however, since reproducible dips in $S$ were observed in repeated experiments only for $x \geqslant 0.20$ and not for the other samples. The origin of the anomaly is not known. Except for the dips, all studied properties of the samples with $x \geqslant 0.20$ (e.g. $S(290 \mathrm{~K}$ ), $\rho(290 \mathrm{~K}), T_{\mathrm{c}}, T^{\max }$, and BVS calculations) are in agreement with the trends expected from the results for $x<0.20$.

The doping concentration dependence of the room temperature $S(290 \mathrm{~K})$ and resistivity $\rho(290 \mathrm{~K})$ are

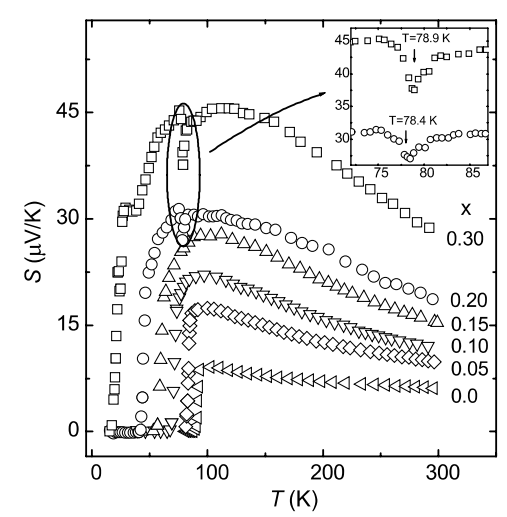

Fig. 1. Thermoelectric power $S$ as a function of temperature and $\operatorname{Pr}$ doping for $\mathrm{Nd}_{1-x} \operatorname{Pr}_{x} \mathrm{Ba}_{2} \mathrm{Cu}_{3} \mathrm{O}_{7-\delta}$. Inset: $S(T)$ in the temperature range $72-87 \mathrm{~K}$, where an anomaly has been observed in the form a dip, for $x=0.20$ and 0.30 .

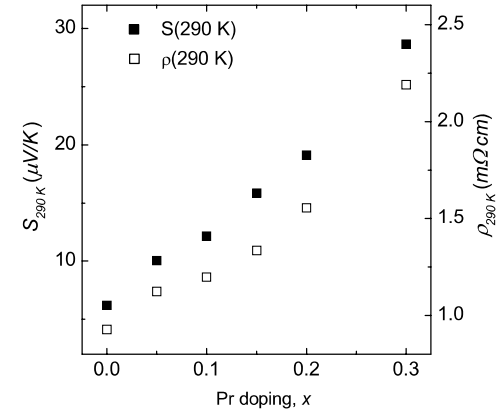

Fig. 2. $S(290 \mathrm{~K})$ (left scale) and $\rho(290 \mathrm{~K})$ (right scale) vs. Pr doping, $x$.

plotted in Fig. 2. Both $S(290 \mathrm{~K})$ and $\rho(290 \mathrm{~K})$ show a continuous increase with increasing $\mathrm{Pr}$ doping. The results for $S(290 \mathrm{~K}), T^{\max }$, and $\rho(290 \mathrm{~K})$ suggest that $\operatorname{Pr}$ reduces hole concentration in the $\mathrm{CuO}_{2}$ plane. This is well in agreement with BVS calculations from neutron diffraction data [5].

\section{Acknowledgements}

We would like to thank I. Bryntse and M. Valldor, Stockholm University, for help with sample preparation and X-ray analysis. Financial support from the Swedish Agencies Vetenskapsrådet and the SSF Oxide Consortium, and from the Iranian Ministry of Science, Research, and Technology are gratefully acknowledged.

\section{References}

[1] Y. Dalichaouch, M.S. Torikachvili, E.A. Early, B.W. Lee, C.L. Seaman, K.N. Yang, H. Zhou, M.B. Maple, Solid State Commun. 65 (1988) 1001.

[2] J.J. Neumeier, T. Bjørnholm, M.B. Maple, I.K. Schuller, Phys. Rev. Lett. 63 (1989) 2516.

[3] A. Kebede, C.S. Jee, J. Schwegler, J.E. Crow, T. Mihalisin, G.H. Myer, R.E. Salomon, P. Schlottmann, M.V. Kuric, S.H. Bloom, R.P. Guertin, Phys. Rev. B 40 (1989) 4453.

[4] G.Y. Guo, W.M. Temmerman, Phys. Rev. B 41 (1990) 6372; R. Fehrenbacher, T.M. Rice, Phys. Rev. Lett. 70 (1993) 3471 ;

A.I. Liechtenstein, I.I. Mazin, Phys. Rev. Lett. 74 (1995) 1000.

[5] S.R. Ghorbani, M. Andersson, Ö. Rapp, Phys. Rev. B, submitted for publication.

[6] Z. Tomkowicz, Physica C 320 (1999) 173.

[7] H.B. Blackstead, J.D. Dow, D.B. Chrisey, J.S. Horwitz, M.A. Black, P.J. McGinn, A.E. Klunzinger, D.B. Pulling, Phys. Rev. B 54 (1996) 6122.

[8] Z. Zou, J. Ye, K. Oka, Y. Nishihara, Phys. Rev. Lett. 80 (1998) 1074.

[9] K.E. Johansson, P.E. Werner, J. Phys. E 13 (1989) 1289. 